\title{
Existe associação entre o diagrama da parestesia da mão e a eletroneuromiografia no diagnóstico da Síndrome do Túnel do Carpo?*
}

\section{Is there an association between the Hand Diagram and Electrodiagnostic Testing for Carpal Tunnel Syndrome?}

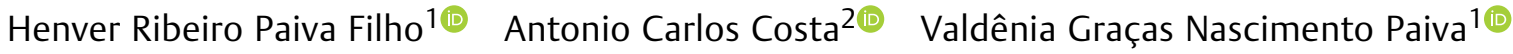 \\ Diego Abad Santos ${ }^{2}$ Ivan Chakkour ${ }^{20}$ \\ ${ }^{1}$ Departamento de Ortopedia, Serviço de Cirurgia da Mão, Hospital de \\ Clínicas, Universidade Federal do Triângulo Mineiro, Uberaba, MG, Brasil \\ ${ }^{2}$ Departamento de Ortopedia, Serviço de Cirurgia da Mão, Santa Casa \\ de São Paulo, São Paulo, SP, Brasil \\ Endereço para correspondência Valdênia das Graças Nascimento \\ Paiva, PhD, Departamento de Ortopedia e Traumatologia, Hospital de \\ Clínicas, Universidade Federal do Triângulo Mineiro, Avenida Getúlio \\ Guaritá, 130, Nossa Sra. da Abadia, Uberaba, MG, Brasil \\ (e-mail: vallfmtm@yahoo.com.br).
}

Rev Bras Ortop 2021;56(1):74-77.

\section{Resumo}

\section{Palavras-chave}

- eletromiografia

- neuropatia mediana

- síndrome do túnel do carpo

Abstract
Objetivo Verificar se existe associação entre o diagrama da parestesia da mão (DPM) e os resultados da eletroneuromiografia (ENMG) no diagnóstico da síndrome do túnel do carpo. Métodos Um total de 92 pessoas preencheram um desenho esquemático da mão com o local exato da parestesia (167 mãos). O autor principal classificou os diagramas de acordo com os critérios de Katz et al. Os resultados do DPM foram cruzados com os resultados positivos da ENMG para o diagnóstico da síndrome do túnel do carpo.

Resultados O padrão possível do DPM foi prevalente tanto isoladamente quanto após o cruzamento com os graus da ENMG.

Conclusão Não houve associação entre o DPM e a ENMG no diagnóstico da síndrome do túnel do carpo.

Objective Verify if there is an association between the hand diagram of paresthesia (HDP) and the results of electroneuromyography (ENMG) in the diagnosis of carpal tunnel syndrome.

Methods A total of 92 people filled in a schematic drawing of the hand with the exact location of the paresthesia (167 hands). The main author classified the diagrams

\footnotetext{
Trabalho feito no Serviço de Cirurgia da Mão da Santa Casa de São Paulo, São Paulo, SP e da Universidade Federal do Triângulo Mineiro, Uberaba, MG, Brasil.
}

recebido

25 de Outubro de 2019

aceito

15 de Abril de 2020

Publicado online

Setembro 25, 2020
DOI https://doi.org/ 10.1055/s-0040-1713763. ISSN 0102-3616.

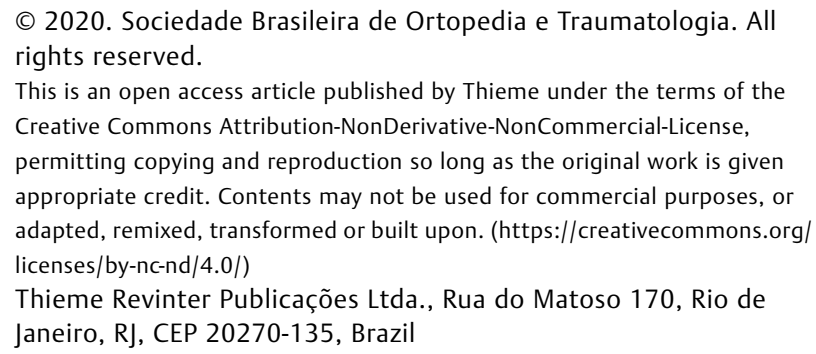

(c) 2020. Sociedade Brasileira de Ortopedia e Traumatologia. All rights reserved.

This is an open access article published by Thieme under the terms of the Creative Commons Attribution-NonDerivative-NonCommercial-License, permitting copying and reproduction so long as the original work is given appropriate credit. Contents may not be used for commercial purposes, or adapted, remixed, transformed or built upon. (https://creativecommons.org/ licenses/by-nc-nd/4.0/) Thieme Revinter Publicações Ltda., Rua do Matoso 170, Rio de Janeiro, RJ, CEP 20270-135, Brazil 
Existe associação entre o diagrama da parestesia da mão e ENMG no diagnóstico da STC Paiva Filho et al. 75

according to the criteria of Katz et al. ${ }^{5}$ The results of the HDP were crossed with the positive results of ENMG for the diagnosis of carpal tunnel syndrome.

Keywords

- electromyography

- median neuropathy

- carpal tunnel syndrome
Results The possible pattern of the HDP was prevalent both in isolation and after crossing with the degrees of ENMG.

Conclusion There was no association between the HDP and ENMG in the diagnosis of carpal tunnel syndrome.

\section{Introdução}

A síndrome do túnel do carpo (STC) é a neuropatia periférica mais comum dos membros superiores decorrente da compressão do nervo mediano no nível do túnel do carpo. ${ }^{1} \mathrm{O}$ diagnóstico geralmente é feito por meio da história clínica e exame físico, ${ }^{2}$ enquanto a eletroneuromiografia (ENMG) auxilia na confirmação diagnóstica dos casos duvidosos e

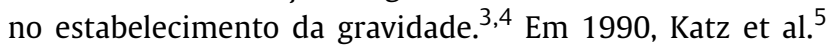
popularizaram os diagramas da mão, que são desenhos esquemáticos nos quais o paciente marca o local exato dos sintomas de dormência nas mãos. Estes diagramas da parestesia da mão (DPMs) foram considerados, à época, como método de triagem em pessoas com suspeita de STC. ${ }^{5,6}$

Em nossa prática clínica e na área da medicina do trabalho, temos observado aumento do número de afastamentos laborais pela STC devido a alguns médicos priorizarem a ENMG positiva para esta afecção em detrimento do exame clínico bem realizado, gerando consequências ao empregador, desde imagem negativa da empresa até atraso na produção, e ao empregado, com cirurgias desnecessárias e afastamentos prolongados. Justificamos a realização do presente trabalho, também, devido à escassez de publicações na literatura que comparem diretamente a classificação do DPM com a ENMG.

O objetivo do nosso estudo foi verificar se existe associação entre o DPM e a ENMG no diagnóstico da STC.

\section{Casuística e Métodos}

Foi realizado estudo transversal, qualiquantitativo, com avaliação de 107 pacientes agendados de forma consecutiva no período de 4 meses. Todos os procedimentos foram realizados de acordo com os padrões éticos determinados pelo Comitê de Ética em Pesquisa para pesquisa em seres humanos e com a Declaração de Helsinque de 1964. O Termo de Consentimento Livre e Esclarecido foi obtido de todos os participantes do estudo, mediante assinatura de termo específico.

Os critérios de inclusão foram pessoas de ambos os gêneros, $>18$ anos de idade, que apresentavam, na consulta inicial, ENMG de membros superiores positiva para STC. Os critérios de exclusão foram pessoas que apresentavam neuropatia da coluna cervical, do nervo mediano na região proximal ou associada ao nervo ulnar, variações anatômicas identificadas pela ENMG, história de polineuropatias, traumatismos ou cirurgias prévias no punho, comorbidades não controladas (diabetes, hipotireoidismo, artrite reumatoide, lúpus eritematoso sistêmico, gota, amiloidose, insuficiência real crônica), tenossinovites ou tumores no punho, gestantes, e pessoas com relatos de atividades laborativas com movimentos repetitivos de flexoextensão dos punhos, vibração de mãos e braços ou exposição laboral ao frio.

No dia e hora agendados da consulta médica, todos os pacientes passaram por triagem realizada por um único enfermeiro com especialização em ortopedia e pertencente ao corpo clínico da universidade que, para o presente estudo, direcionou para nossa equipe as pessoas que apresentavam ENMG dos membros superiores positiva para STC.

Em seguida, assim que os pacientes entravam no consultório médico, antes da consulta, foram orientados sobre o preenchimento do desenho esquemático das mãos. Esta orientação foi realizada por um único médico membro da equipe que não tinha conhecimento de quaisquer dados do participante. $O$ preenchimento consistiu na marcação em caneta da região correspondente à localização exata da parestesia. 0 nosso estudo abordou apenas a localização da área marcada no DPM, e não a intensidade dos sintomas. Após o término do preenchimento, o autor principal do presente trabalho classificou o diagrama segundo os critérios de Katz et al., ${ }^{5}$ sem conhecimento de quaisquer dados clínicos do paciente estudado.

Katz et al., ${ }^{5}$ em 1990, propuseram um diagrama da mão, no qual a localização da parestesia é marcada por meio de desenhos esquemáticos. Classificaram os resultados como clássico, provável, possível ou improvável.

Após o preenchimento do DPM, o desenho foi guardado em arquivo próprio para posterior análise estatística e, então, o participante foi submetido ao atendimento médico conforme rotina ambulatorial, momento o qual foram obtidas as informações das variáveis qualiquantitativas.

A ENMG foi realizada pelo mesmo médico neurologista especializado em estudos eletroneuromiográficos, pertencente ao corpo clínico institucional, cego para os dados clínicos e sem vínculo específico com o presente trabalho. 0 exame é realizado bilateralmente, conforme normatização institucional local. Foi utilizado o eletroneuromiógrafo do modelo Neuropack EMG S1, MEB-9400K (Nihon Kohden Corporation, Tóquio, Japão), em temperatura da sala de $28^{\circ} \mathrm{C}$, disponível em nossa instituição. A classificação da ENMG é assim padronizada: grau leve (alteração somente da condução sensitiva), grau moderado (alteração da condução sensitiva e motora) e grau grave (condução sensitiva e motora alteradas e sinais de denervação à eletromiografia de agulha).

As informações obtidas dos participantes foram organizadas em um banco de dados, conforme os critérios de Katz et al. ${ }^{5}$ (clássico, provável, possível ou improvável) para STC, e conforme a gravidade da ENMG (leve, moderada ou grave). 
Diagrama da parestesia da mão - DPM

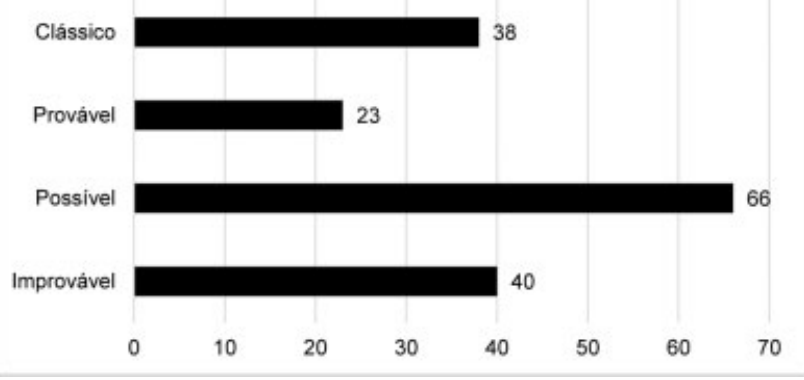

Fig. 1 Distribuição do número de mãos de acordo com o diagrama da parestesia da mão.

Os resultados do DPM foram cruzados com o resultado positivo da ENMG para STC, utilizando a mão como unidade amostral, com o uso de teste da razão de verossimilhanças. ${ }^{7}$

Foram atendidas 107 pessoas e excluídas 15 (1 gestante, 2 com traumatismo prévio no punho, 5 com diabetes e/ou hipotireoidismo não controlados e 7 com cirurgias prévias no punho), totalizando 92 participantes incluídos no presente estudo. Em relação ao gênero, nossa amostra apresentou 14 homens $(15,2 \%)$ e 78 mulheres $(84,8 \%)$. A média e desvio padrão (DP) da idade foi 55,2 $\pm 10,5$ anos.

Dos 92 pacientes com ENMG positiva para STC, e utilizando a mão como unidade amostral, obtivemos 75 pessoas com ENMG positiva bilateralmente (150 mãos) e 17 unilateralmente (17 mãos), totalizando 167 mãos com resultado positivo da ENMG para STC.

Para a realização das análises, foi utilizado o software IBM SPSS Statistics for Windows, Versão 20.0 (IBM Corp., Armonk, NY, EUA) e para tabulação dos dados foi utilizado o software Microsoft Excel 2003 (Microsoft Corporation, Redmond, WA, EUA). Os testes foram realizados com nível de significância de $5 \%$.

\section{Resultados}

A - Figura 1 mostra a distribuição das 167 mãos que apresentavam ENMG positiva para STC, classificadas de acordo com os critérios de Katz et al. ${ }^{5}$ Em nosso estudo, obtivemos 38 mãos (22,7\%) com padrão clássico, 23 (13,8\%) provável, 66 (39,5\%) possível e 40 (24,0\%) improvável.
A - Tabela 1 apresenta o cruzamento entre o DPM e a ENMG, com os resultados estatísticos. Em ordem decrescente de acometimento, observamos que das 22 mãos com ENMG grau leve, 10 casos (45,5\%) apresentaram DPM padrão possível, seguida por improvável em 6 casos (27,2\%), clássico em 4 casos $(18,2 \%)$ e provável em 2 casos (9,1\%). Em ordem decrescente de acometimento, observamos que das 67 mãos com ENMG grau moderado, 26 casos (38,8\%) apresentaram DPM padrão possível, seguida por clássico em 18 casos (26,9\%), improvável em 16 casos (23,9\%) e provável em 7 casos $(10,4 \%)$. Por fim, em ordem decrescente de acometimento, observamos que das 78 mãos com ENMG grau grave, 30 casos (38,5\%) apresentaram DPM padrão possível, seguida por improvável em 18 casos (23,1\%), clássico em 16 casos (20,5\%) e provável em 14 casos (17,9\%).

\section{Discussão}

Atualmente, contamos com diversos instrumentos clínicos para o diagnóstico da STC, mas é essencial que estejam validados. ${ }^{8}$ Concordamos com Moradi et al. ${ }^{9}$ ao considerarem o DPM como método de rastreio para o diagnóstico da STC, inclusive utilizado pela nossa equipe na abordagem inicial. Das 167 mãos avaliadas em nosso trabalho, identificamos no DPM que o padrão possível foi o mais prevalente, com 66 casos (39,5\%), enquanto o menos prevalente foi o padrão provável, em 23 casos (13,8\%).

Observamos que existem divergências de opinião sobre a necessidade da ENMG para a confirmação diagnóstica da STC, e a literatura é controversa ao demonstrar qual é o melhor método diagnóstico. A concordância entre sintomas, exame físico e ENMG é baixa, tendo Stevens encontrado concordância de $5 \%$ entre os 3 parâmetros. ${ }^{10}$ Estes autores acreditam que a pessoa com ENMG positiva para a STC na ausência de sintomas não pode ser considerada como portadora da STC.

Quando avaliamos o cruzamento entre DPM e ENMG, observamos que tanto nos casos com ENMG grau leve, moderado ou grave houve predominância do DPM padrão possível. Por outro lado, o padrão provável foi o menos prevalente em todos os graus de acometimento da ENMG. Em nossos resultados, verificamos que não houve associação de significância estatística $(p=0,797)$ entre o DPM e os resultados da ENMG.

Tabela 1 Cruzamento entre o diagrama da parestesia da mão e a eletroneuromiografia

\begin{tabular}{|c|c|c|c|c|c|}
\hline \multirow[t]{2}{*}{ DPM } & \multicolumn{3}{|l|}{ ENMG } & \multirow[t]{2}{*}{ Total } & \multirow[t]{2}{*}{ valor-p } \\
\hline & Leve & Moderada & Grave & & \\
\hline Clássico & $4(18,2)$ & $18(26,9)$ & $16(20,5)$ & $38(22,8)$ & \multirow[t]{4}{*}{0,797} \\
\hline Provável & $2(9,1)$ & $7(10,4)$ & $14(17,9)$ & $23(13,8)$ & \\
\hline Possível & $10(45,5)$ & $26(38,8)$ & $30(38,5)$ & $66(39,5)$ & \\
\hline Improvável & $6(27,2)$ & $16(23,9)$ & $18(23,1)$ & $40(24,0)$ & \\
\hline Total & $22(100)$ & $67(100)$ & $78(100)$ & $167(100)$ & \\
\hline
\end{tabular}

Abreviações: DPM, Diagrama da Parestesia da Mão; ENMG, Eletroneuromiografia.

Teste da razão de verossimilhanças. 
Como pontos positivos do nosso estudo, tivemos casuística com 92 pacientes, totalizando 167 mãos. Os estudos de Sharma et al. ${ }^{11}$ incluem 40 pacientes e 71 mãos; Nelson et al., ${ }^{12} 26$ pacientes e 34 mãos; e Katz et al., ${ }^{5} 63$ pacientes e 85 mãos. Ademais, a ENMG foi realizada somente por um médico neurologista especializado, utilizando um único aparelho de ENMG disponível em nossa instituição. Como ponto negativo, é possível que tenha ocorrido o viés referente à classificação do DPM que, em nosso estudo, foi realizado por um único médico da equipe. Por ser instrumento de avaliação subjetiva, uma das medidas para diminuir esse viés seria a classificação dos diagramas por dois ou mais médicos.

Não encontramos na literatura, até o momento do presente estudo, recomendações, diretrizes ou critérios suficientes e validados a nível nacional de quando a ENMG deverá ser solicitada para investigação da STC, o que torna o diagnóstico dessa afecção desafiador.

Entendemos que é temerário excluir o diagnóstico da STC em pessoas com sintomas típicos e ENMG normal, até porque não há como prever se a pessoa está apresentando isquemia transitória pois, neste caso, a ENMG pode estar normal. Acreditamos que a ENMG é um exame útil nos casos duvidosos e refratários ao tratamento inicial, devendo ser solicitada de forma criteriosa e com embasamento naquilo que se pretende investigar, evitando-se o desconforto do exame e a ansiedade do paciente. Em relação ao DPM, acreditamos que mais estudos são necessários para comprovar a eficácia e a precisão deste método para o diagnóstico da STC, porém entendemos que esta ferramenta clínica pode ser utilizada para rastreio dessa afecção.

Em nossa opinião, nem o DPM, tampouco a ENMG, devem ser utilizados isoladamente ou em substituição da história clínica pormenorizada e exame físico minucioso para o diagnóstico da STC.

Concluímos que não existe associação entre o DPM e a ENMG no diagnóstico da STC.
Conflito de Interesses

Os autores declaram não haver conflito de interesses.

\section{Referências}

1 Spahn G, Wollny J, Hartmann B, Schiele R, Hofmann GO. [Metaanalysis for the evaluation of risk factors for carpal tunnel syndrome (CTS) Part I. General factors]. Z Orthop Unfall 2012;150 (05):503-515

2 Wang WL, Buterbaugh K, Kadow TR, Goitz RJ, Fowler JR. A prospective comparison of diagnostic tools for the diagnosis of carpal tunnel syndrome. J Hand Surg Am 2018;43(09):833-836.e2

3 Rempel D, Evanoff B, Amadio PC, et al. Consensus criteria for the classification of carpal tunnel syndrome in epidemiologic studies. Am J Public Health 1998;88(10):1447-1451

4 Sears ED, Swiatek PR, Hou H, Chung KC. Utilization of preoperative electrodiagnostic studies for carpal tunnel syndrome: an analysis of National Practice Patterns. J Hand Surg Am 2016;41(06): 665-672.e1

5 Katz JN, Stirrat CR. A self-administered hand diagram for the diagnosis of carpal tunnel syndrome. J Hand Surg Am 1990;15 (02):360-363

6 Calfee RP, Dale AM, Ryan D, Descatha A, Franzblau A, Evanoff B. Performance of simplified scoring systems for hand diagrams in carpal tunnel syndrome screening. J Hand Surg Am 2012;37(01): $10-17$

7 Kirkwood BR, Sterne JA. Essential medical statistics. $2^{\text {nd }}$ ed. Blackwell ScienceMassachusetts, USA2006:502

8 Goulart BNG, Chiari BM. [Screening versus diagnostic tests: an update in the speech, language and hearing pathology practice]. Pro Fono 2007;19(02):223-232

9 Moradi A, Mellema JJ, Oflazoglu K, Isakov A, Ring D, Vranceanu AM. The relationship between catastrophic thinking and hand diagram areas. J Hand Surg Am 2015;40(12):2440-6.e5

10 de Krom MC, Kester AD, Knipschild PG, Spaans F. Risk factors for carpal tunnel syndrome. Am J Epidemiol 1990;132(06):1102-1110

11 Sharma V, Wilder-Smith EP. Self-administered hand symptom diagram for carpal tunnel syndrome diagnosis. J Hand Surg $[\mathrm{Br}]$ 2004;29(06):571-574

12 Nelson JT, Gauger MR, Whaley JD, Zinberg EM. Patient-reported symptom-mapping in carpal tunnel syndrome. Muscle Nerve 2019;59(03):321-325 